\title{
Research on Cost Allocation Model of Telecom Infrastructure Co-construction Based on Value Shapley Algorithm
}

\author{
YeYuan ${ }^{1}$, Haigang Sheng ${ }^{1}$ and Haohao $\mathrm{Shi}^{1}$ \\ ${ }^{1}$ Chongqing University of Posts and Telecommunications Chongqing, 400065, \\ China \\ Email:914867023@qq.com,1533455810@qq.com,1103745171@qq.com
}

\begin{abstract}
Under the circumstance that China Tower unified construction of $4 G$ telecommunications infrastructure, how do the three operators fairly and reasonably share the telecommunications infrastructure cost is the key problem of Chinese telecom industry at present, and also is a hot issue for scholars. To alleviate this problem, after comparing the advantages and disadvantages of current methods of cost allocation, this paper try to introduce risk correction factor into Shapley Value algorithm, and propose a cost allocation model of telecom infrastructure co-construction based on the fixed Shapley Value algorithm. The validity and practicability of the method have been proved by the test of the actual calculation. With the help of this model, it is able to provide a more effective strategy for the cost allocation problem in the construction of China's telecom infrastructure.
\end{abstract}

Keywords: Fixed ShapleyValue algorithm; Cost allocation; Risk factors; telecom infrastructure

\section{Introduction}

In China, it is a major problem in co-construction and sharing work that how to share the cost of telecommunications infrastructure fairly and reasonably for the three telecom operators. With the introduction of "Broadband China", "Improvement Project of Small and Medium-sized City Infrastructure Network" and a series national strategy of network infrastructure construction, the competition for network infrastructure resources of telecom operators is becoming more and more intense. Meanwhile, in the sharing work of CPN access, due to the local operator's intense competition and some historical reasons, the progress of telecommunications infrastructure sharing has been very slow. Although the relevant state ministries and the Ministry of Industry and Information Technology have successively introduced the "Emergency Notice on Promoting the Sharing of Telecommunications Infrastructure","Interim Provisions on Sharing of Telecommunications Infrastructure Engineering and Technical" and other policy documents, which make clear provisions on the principle of sharing, technical scheme and so on. While in terms of co-construction cost sharing, these documents just point out that co-construction cost should be shared by the cost and don't clear the specific details, which causes the difficulties in the implementing of co-construction cost of telecommunications infrastructure and hinders the smooth progress of the work sharing to some extent. It has become an important issue needed to clear or solve addressed in the current sharing work how to share the telecommunications infrastructure costs fairly and reasonably to improve sharing enthusiasm of three operating companies. 


\section{The Main Cost Allocation Method and its Application}

Now, in all walks with the continuous development of co-construction and sharing level, investment cost allocation problem has also been a considerable degree of attention in theory. It has formed dozens of cost allocation methods, but these methods can be summarized in the following categories: one-time allocation method, the two-time allocation method and allocation method based on Cooperative Game.

The one-time allocation method means that makes one-time allocation of costs according to the proportion of a quantitative index of the project, mainly based on the participating parties to allocate averagely. However, this method did not take into account the complexity of the co-construction process, and treated the various different companies fully equally, so it is a relatively unfair method.The two-time allocation methodrefers to that the investment of the project is divided once, and then on the basis of division,the investment is allocated by proportion. The two-time allocation method refers primarily to the Separable Cost--The Separable Costs-remaining Benefits Method (SCRB). In this method, firstly separable investment (minimum investment required by each unit) of benefit units will separated from total cost of the project. Then according to proportion of surplus benefit of each beneficiary unit, remaining investment will be allocated. The twotime allocation method refers primarily to the Separable Cost - The SeparableCostsremaining Benefits Method (SCRB). In this method, firstly separable investment (minimum investment required by each unit) of benefit units will separated from total cost of the project. Then according to proportion of surplus benefit of each benefit unit (the smaller value of each benefit unit and the equivalent alternative investment scheme), remaining investment will be allocated. Because these two methods have great limitations, it is not widely used in practice.

Cost allocation can be regarded as a typical cooperative game process. In the process of cooperative game, participants not only consider their own interests, to be able to enter the next round of the game and gain further common interests, the participants will also consider the overall interests of the game groups. Cooperative game has a wide range of applications in the cost allocation, such as Anily and Haviv (2010) use the core concept of cooperative game to study the cooperative cost allocationof $\mathrm{M} / \mathrm{M} / 1$ queuing system with shared resources and customers at the same time;Wang Wei (2011) on the basis of cooperative game theory and the studies of other scholars, in the aspect of joint distribution in improving the logistics benefit, use a combination of qualitative and quantitative methods to discuss the income distribution and cost problems of joint distribution, which makes up for the shortcomings of some of the literature.

2012 Nobel Prize winner Shapley Lloyd and others make a great contribution on the cooperative game, which put forward a series of important theories, such as BondarevaShapley rule, Shapley-Shubik. At present, many studies on cooperative game are around Shapley value method to expand, such as Reinhardt and Dada use the Shapley value of cooperative games to study the cooperative cost allocation of queuing system with shared resources and customers at the same time; Li Weiqian et al. (2013), based on the DEA method, improved the value of Shapley, which provides a reference for the study of the ecological compensation allocation of the trans regional watershed; Zeng Yinlian and $\mathrm{Li}$ Jun, in the study on cooperation cost allocation problem of queuing system with shared resources, verified that the allocation method based on the core is superior than the Shapley value allocation method and the proportional distribution method by simulation analysis.

According to the related research of cost allocation in academic circles at present, in the cost allocation method, based on cooperative game the cost allocation method is relatively more common, and the most widely used is Shapley value method, which distribution principle is relatively more equitable. And at present, in the study of telecommunications cost allocation, Liu Xiaoning (2014) using activity-based costing, 
Tang Wei, etc. (2007) using allocation cost based on life-cycle passenger flow are the main representatives. Meanwhile, the cost allocation theory based on cooperative game has been more applied in the cost allocation problems about some large public infrastructure, such as Choudhury(2011)studied cost allocation problems in the field of electric power communication network. However, based on the Shapley value method the present studies of telecommunications infrastructure cost allocation are not yet abundant. In view of this, this paper introduces the Shapley value method to study the cost allocation of telecom infrastructure sharing and puts forward cost allocation strategy, which makes Shapley value method and risk factor as correction.

\section{Basic Principle of Shapley Value Algorithm}

Shapley Value Algorithm method which is proposed by Shapley L.S. in 1953is a mathematical method to solve the problem of multi-person cooperation. It is mainly used to solve distribution of profits, cost allocation and other problems. It is a way to allocate resources according to the contributions of alliance members, which reflects the fairness and reasonableness. And it can improve the team members' actualityof participation. For cost allocation in this research, special allocation theory is following:

Let characteristic function is a co-constructioncost function of all sets defined on the set of co-construction and operation enterprise. The co-construction and operation enterprise set is $N=\{1,2,,,,,, n\}$. For any possible set $\mathrm{S}(\mathrm{S}$ is a subset of N.), which iscoconstructiontelecom operators set to build jointly, there will produce a total cost $\mathrm{C}(\mathrm{S})$ produced by thealliance.

Let $^{N}=\{1,2,,,,,,, n\}, \mathrm{C}(\mathrm{S})$ is defined on the function of all subsets of $\mathrm{N}$, and satisfies the following conditions:

$$
\begin{gathered}
\mathrm{C}(\phi)=0 \\
C(N) \leq \sum_{i=1}^{n} C(i)
\end{gathered}
$$

Among them, $(\mathrm{N}, \mathrm{C})$ means the cooperative game of $\mathrm{n}$ telecom operators. $\mathrm{C}(\mathrm{S})$ is said the characteristic function of cost to co-construction. $\mathrm{C}(\mathrm{N})$ is the total cost of $\mathrm{n}$ enterprises to co-construction. $C(i)$ is the cost of NO $i_{\text {enterprise to build separately. The equation }}$ (1) expresses that when the set is empty(no one carries out to build jointly), the coconstruction cost is zero, which is quite obvious.The equation (2) reflectscollective rationality ofalliance, which means that the cost ofco-construction is lesser than the sum of all costs to build separately. Otherwise, it is obvious that telecom operators are unnecessary to participate in co-construction.

For every telecom operator participating in co-construction, they should share their own cost in the total cost. It is expressed by $x=\left(x_{1},,,, x_{n}\right)$, where ${ }^{x_{i}}$ is the cost that NO

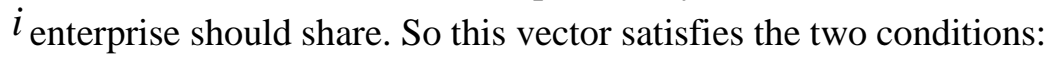

$$
\begin{gathered}
x_{i} \leq C(i) i \in N \\
\sum_{i=1}^{n} x_{i}=C(N)
\end{gathered}
$$

${ }^{x_{i}}$ meansone enterprise's allocation cost, andthe set of all $x$ constitutes sharing set. The condition (3) is individual reason conditionthat the cost allocation of NO $i$ enterprise is lesser than the cost to build separately by itself. The condition (4) iseffective conditionthat the sum of cost allocation of $n$ enterprises is the total cost to build jointly. It is clear that the element of sharing set is not the only. And when cost allocation to coconstructionhas been solved, a more reasonable sharing result should be pointed out. 
From Shapley Value model law, it will share the cost of adding value which is benefitedby the co-construction of telecom enterprises. And drawout the cost allocation of NO. $i_{\text {enterprises. }}$

$$
x_{i}=\frac{(|s|-1) !(n-|s|) !}{n !} \sum[C(s)-C(s \backslash i)]
$$

Where, $|s|$ is the number of members in the alliance S. $C(s)-C(s \backslash i)$ is the increased cost caused by NO $i$ enterprise joining in the alliance $\mathrm{S}$, that is the marginal cost of NO.

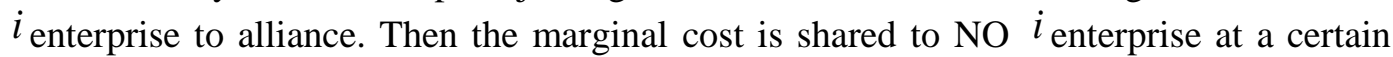
probability
undertake.

As can be seen from the conclusion above, Shapley Value Algorithm shares the cost by the size of contribution of every enterprise, which satisfies individual reason and effectiveness. Besides, it reflects "fairness" and "reasonableness" to a certain extent,and it can encourage the participating enterprises make greater contribution in cooperation.

\section{The Correction of Shapley Value and the Meaning of Correction}

\subsection{The Correction of Shapley Value}

As can be seen from the analysis above, using Shapley Value to allocate thecost allocation with doing dynamicalliance avoids inequitable division, and it reflects fairness and reasonableness to a certain extent. But in the telecominfrastructure to co-construction, for every telecom operators, the extent of cooperation and the strength of participation are different. Especially, the risks undertaken in the process to co-construction of operators are different. For the operators who undertake greater risk, the cost allocation should be lesser. For the operators who undertake lower risk, the cost allocation should be more.

Here, let $R$ is the risk factor. If the impact of risk factor is not considered, the risks

that three operators undertake in the process to build jointly are equal, whichis $\bar{R}=\frac{1}{n}$. n is the number of operators to build jointly. Obviously, this is the ideal situation andit is impossible in practice.

Analysis above indicates that, the total cost to co-construction is $\mathrm{C}(\mathrm{N})$. In considering the ideal case of equal risk, the cost allocation of every operator participating in coconstructionis $x_{i}$. In considering the impact of risk factors, let the actual cost allocationis $x_{i}^{\prime}$, the risk undertaken is $R_{i}, i=1,2,,,,, n$. The difference value of $R_{i}$ and $\bar{R}_{\text {is: }}$

$$
\Delta R_{i}=R_{i}-\frac{1}{n}
$$

Among them, $\sum_{i=1}^{n} R_{i}=1 \sum_{i=1}^{n} \Delta R_{i}=0$

The correction of cost allocationis $\Delta x_{i}=C(N) \times \Delta R_{i}$. So in considering all risk factors, actual cost allocation is:

$$
x_{i}^{\prime}=\frac{(|s|-1) !(n-\mid s) !}{n !} \sum[C(s)-C(s \backslash i)]+C(N) \times \Delta R_{i}
$$

When the specific cost is assessed, the principle of contribution is following. 
(1)When $\Delta R_{i} \geq 0$, the actual risk NO. ${ }^{i}$ operatorundertaking is higher than the ideal case in the process to build jointly. So they should share lesser costs. The actual cost allocation of NO. $i_{\text {operator is }} x_{i}^{\prime}=x_{i}-\Delta x_{i}$

(2)Similarly, when $\Delta R_{i}<0$, actual risk NO. ${ }^{i}$ operator undertaking is lower than the ideal case in the process to build jointly. So they should share more costs. The actual cost allocation of NO. $i_{\text {operator is }} x_{i}^{\prime}=x_{i}+\left|\Delta x_{i}\right|$.

\subsection{The Meaning of Correction Factor}

Analysis above indicates that, for telecom infrastructure of three operators to coconstruction, the risk factors mainly include the risk of policy policy, technology risk, cost risk, construction progress risk, management risk and the other risks. The risks of telecominfrastructure to different operators are different.

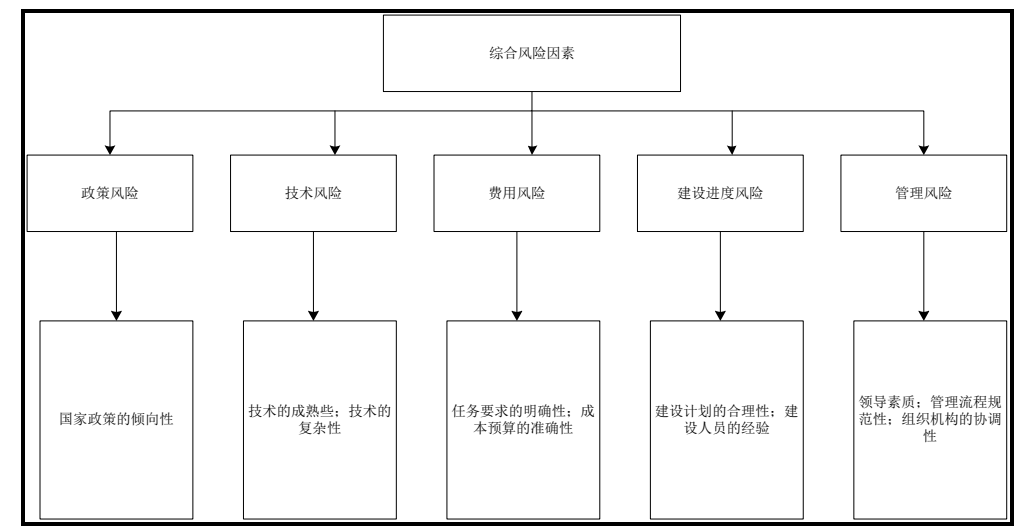

\section{Figure 1. Risk factors for the Construction of Telecommunications Infrastructure}

After clearing the definition of each risk, it is necessary to define the size of risk operators undertaken. Fieldwork, expert counseling method and the other methods are used to determine qualitatively. And fuzzy mathematics, genetic algorithms, neural networks, AHP and the other methods are also can be used to determine. Because the methods of risk factors are not the focus of this study, we do not go into in detail about the risk factors.

\section{Application Examples}

According to the regulation, "The Emergency Notification of Promoting Joint Construction and Sharing of Telecommunications Infrastructure" issued by Ministry of Industry and Information Technology, telecom infrastructure to co-construction mainly includes iron tower, pole line, rooftop, machine room, indoorsignal distributed system, pipeline and so on. In telecom infrastructure to co-construction, taking iron tower as an example, let the costs of iron tower NO. 1, 2, 3 operators to build separately are 10 million, 10 million and 10 million respectively. The cost of NO. 1, 2 operators to coconstructionis 10 million. The cost of NO. 2, 3 operators to co-constructionis 11 million.The cost of NO. 1, 3 operators to co-constructionare 12 million. The cost of NO $1,2,3$ operators to co-construction is 13 million.

According to Shapley Value model above, NO. 1,2,3 operators participating in coconstruction constitute an alliance $\mathrm{N}, N=\{1,2,,,,,, n\}$.According to the above assumptions, we can get that: 


$$
\begin{gathered}
C(\{1\})=10 \quad, \quad C(\{2\})=10 \quad, C(\{3\})=10 \quad, C(\{1,2\})=10 \quad, C(\{2,3\})=11 ， \\
C(\{1,3\})=12, C(\{1,2,3\})=13 .
\end{gathered}
$$

For NO.1 operator, the cost allocation should be calculated with Shapley Value Algorithm,(figure 1)

Table 1.The Cost Allocation of No.1 Operator

\begin{tabular}{|l|l|l|l|l|}
\hline & $\mathbf{( 1 )}$ & $\mathbf{( 1 , 2 )}$ & $\mathbf{( 1 , 3 )}$ & $\mathbf{( 1 , 2 , 3 )}$ \\
\hline$C(s)$ & 10 & 10 & 12 & 13 \\
\hline$C(s \backslash i)$ & 0 & 10 & 10 & 11 \\
\hline$C(s)-C(s \backslash i)$ & 10 & 0 & 2 & 2 \\
\hline$|s|$ & 1 & 2 & 2 & 3 \\
\hline$\frac{(|s|-1) !(n-|s|) !}{n !}$ & $1 / 3$ & $1 / 6$ & $1 / 6$ & $1 / 3$ \\
\hline$\frac{(|s|-1) !(n-|s|) !}{n !} C(s)-C(s \backslash i)$ & $10 / 3$ & 0 & $1 / 3$ & $2 / 3$ \\
\hline
\end{tabular}

Where, $\mathrm{S}$ is the subset of $\mathrm{N}, C(s)$ is the total cost of operators participating in coconstruction in the alliance $\mathrm{S}$. $C(s \backslash i)$ is the total cost of the operators participating in building jointly except NO $i$ operator. $|S|_{\text {is }}$ the number of members in alliance S. $\underline{(|s|-1) !(n-|s|) !}$

$n ! \quad$ is the possible probability of operators to participating in coconstruction, or is called the weighting factor. $C(s)-C(s \backslash i)$ is the marginal cost of NO. ${ }^{i}$ operator to alliance $\mathrm{S}$, that is the size of contribution of NO $i$ operator to alliance $\mathrm{S}$.

The cost allocation of NO.1 operator calculated by adding the last low of Figure 1 is 43,000 Yuan.Similarly, the cost allocations of NO 2, 3 are 38,000 Yuanand 48,000 Yuan separately.If we donot considerthe impact of risk factors, the cost allocations of NO 1,2,3 operators participating in co-construction are calculated, $x_{1}=43,000$ Yuan, $x_{2}=38,000$ Yuan, $X_{3}=48,000$ Yuan. Considering risk factors, it assumes that the risk factors of three operators are calculated $R_{1}=0.2, R_{2}=0.4, R_{3}=0.4$ with AHP. So $\Delta R_{1}=-2 / 15$, $\Delta R_{2}=1 / 15$ and $\Delta R_{3}=1 / 15$.

According to Shapley Value model modified above, it is calculated that $x_{1}^{\prime}=x_{1}+\left|\Delta x_{1}\right|=49,000$ Yuan, $\quad x_{2}^{\prime}=36,000$ Yuan, $\quad x_{3}^{\prime}=45,000$ Yuan.

The sum of these two results (the total build cost) are 130,000 Yuan. It is easy to verify that the cost allocation $x_{i}^{\prime}$ of three operators still meets the basic condition of original Shapley Value after considering risk factors. According to the assumption of risk factor values, it can be seenthat the risk factors of telecom operators 1 are small, and the risk factors of 2 and 3 are relatively large. The results indicate that after adding risk factors, the cost-sharing ofoperators 1 should be increased, and the operators 2 and 3 should be reduced. The above calculation results are consistent with the correction hypothesis. Therefore, after considering risk factors, the cost allocation will be more fair and equitable. 


\section{Conclusion}

In this paper, the Shapley Value model is introduced into the analysis and research of the cost allocation of telecom infrastructure co-construction. Compared with several cost allocation methods, Shapley Value model is more fair and reasonable, which is based on the contribution of telecom operators in the process of co-construction to share the cost. Based on the risk allocation problem in co-construction and sharing of telecom operators, the Shapley Value is modified by introducing risk factors and the cost allocation strategy of Shapley Value is proposed, with correction algorithms as risk factors. The research of this paper has a certain guiding significance for improving the scientific rationality of the sharing of the co-construction cost of telecom operators, and the enthusiasm of the telecom operators to cooperate on building the infrastructure.

\section{Acknowledgement}

This work was supported by Doctoral Fund of Chongqing University of Posts and Telecommunications (K2014-13), Social science planning project of Chongqing city in 2015 (2015YBGL111),2015 National Social Science Foundation of China West Project(15XGL024), and the Chongqing municipal education commission project of humanities and social science, research on accelerate the path of the triple play in Chongqing and policy(16SKGH058)

\section{References}

[1] C. Wei and Z. Yingchun, "Cost Al location Methods Based on Cooperative Games", Operations Research and Management, vol. 13, no. 4, (2004), pp. 54-57.

[2] L. Yongjun, "One Fixed Cost-sharing Method Based on Bargaining Game Between DEA and Nash", System Engineering, vol. 26, no. 6, (2008), pp. 73-77.

[3] W. Wei and Z. J. song, "Based on Cooperative Game Analysis the Distribution of Income and Cost Sharing of the Joint Distribution", Science \& Technology Information, vol. 29, (2011), pp. 131-132.

[4] L. W. Qian, X. J. Cang, L. J. Xun and S. Hai, "Method of allocating ecological compensation amount of river basin based on improved Shapley value", Systems Engineering-Theory \& Practice, vol. 33, no. 1, (2013), pp. 255-261.

[5] Z. Y. Lian and L. Jun, "Cost Allocation for Cooperation in Queuing System with Application of Cooperative Game Theory”, System Engineering, no. 12, (2013).

[6] L. X. Ning, "Research on the establishment and application of activity based costing system in Telecom Enterprises", Western Finance and Accounting, no. 10, (2014), pp. 28-30.

[7] T. Wei, C. S. Fa and Q. X. Yang, "The Urban Rail Transit Pricing Method by Life Cycle Cost Apportioned in the Entire Passengers", Systems Engineering-Theory \& Practice, vol. 27, no. 5, (2007), pp. 69-74.

[8] I. Giannoccaro and P. Pontrandolfolaria, "Supply chain coordination by revenue sharing contracts", International Journal of Production Economics, no. 89, (2004), pp. 131-139.

[9] S. Anily and M. Haviv, "Cooperation in Service Systems", Operations Research, vol. 58, no. 3, (2010), pp. 660-673.

[10] S. Gavirneni, "Benefits of cooperation in a production distribution environment", European Journal of Operational Research, vol. 130, (2001), pp. 612-622.

[11] E. Kadir, "Coordinating supply chain in decentralized environments: optimization auction and bargaining-theoretic models", Lehigh University, (2002).

[12] G. Reinhardt and M. Dada, "Allocating the gains from resource pooling with the Shapley Value", Journal of the Operational Research Society, vol. 56, no. 8, (2005), pp. 997-1000.

[13] N. B. D. Choudhury, S. K. Goswami, "Artificial intelligence solution to transmission loss allocation problem”, Expert Systems with Applications, vol. 38, no. 4, (2011), pp. 3757-3764. 


\section{Authors}
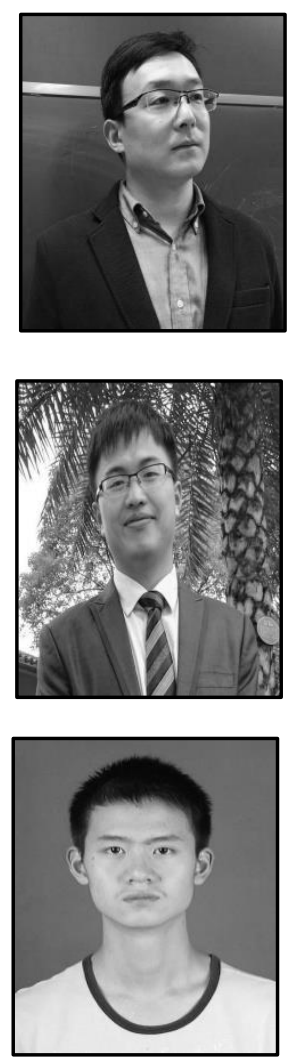

Ye Yuan, he is an instructor of Management Science in School of Economics and Management at Chongqing University of Posts and Telecommunications. His main research interests are next generation network, consumer behaviour and management of science.

HaigangSheng, he is a graduate student in Chongqing University of Posts and Telecommunications, research direction for the management of science and Engineering.

HaohaoShi, he is an undergraduate student in Chongqing University of Posts and Telecommunications, research direction for marketing management and consumer behavior. 